\section{Establishment of morphine preference in the rat*}

KHALIL A. KHAVARI and MARC E. RISNER

The University of Wisconsin-Milwaukee, Milwaukee, Wis. 53201

Three groups of rats were placed on sucrose morphine and milled rat food for 25 days. Morphine $\mathrm{HCl}$ content was $.5 \mathrm{mg} / \mathrm{ml}$ for all three groups. Sucrose concentrations were $2.5 \%, 5 \%$, and $10 \%$. In a subsequent choice test, all three groups selected their respective sucrose morphine over tap water. In an additional choice test given to the $10 \%$ group, the rats selected the sucrose morphine over the vehicle. We report on a highly effective oral method of inducing morphine preference.

There is a general agreement that drug research with infrahuman organisms can be of potential value for understanding the mechanisms of dependence in man. Commonly employed procedures for studies of dependence in animals involve chronic administration of drugs via intraperitoneal, subcutaneous, and intravenous routes. Although the oral method of drug administration is the simplest, most economical, and used most frequently in human medications, this procedure has not been widely employed in animal research (Schuster \& Thompson, 1969). In the case of opiates, a few investigators (e.g., Thompson \& Ostlund, 1965; Stolerman \& Kumar, 1970), emplying the oral method of morphine administration, have successfully produced drug dependence in the rat. However, since morphine is a bitter substance, rats must be forced to drink morphine solutions. We now report data that show that rats ingest high quantities of morphine when the drug is presented in a sucrose medium and that they prefer sucrose morphine to sucrose alone.

\section{METHOD}

The Ss were 11 male Holtzman Sprague-Dawley rats, 170-190 days old at the start of the experiment. Each rat was individually housed in a standard laboratory cage with free access to milled Wayne rat food. In addition, each rat was provided with a free source of liquid which contained $.5 \mathrm{mg}$ of morphine $\mathrm{HCl}$ in every milliliter. The solution vehicles were $2.5 \%$ sucrose water for three rats, $5 \%$ sucrose water for four rats, and $10 \%$ sucrose water for four rats. After 25 days of the above regimen, the three groups were presented with a choice test. That is, each group was given access to free food and their respective

*Supported in part by NSF Research Grant B023365 and NIMH Research Grant 16748-01 to K. A, Khavari. sucrose-adulterated morphine solution in addition to free water. This situation was maintained for 13 consecutive days for all three groups. Daily measures of body weight and of liquid and food intake were taken. In order to guard against position preference, the location of water and sucrose morphine sources was determined randomly from day to day for each rat.

Beginning with Day 14 of the choice test and continuing through Day 22, a new manipulation was introduced for the group that had originally been maintained on $.5 \mathrm{mg}$ of morphine $\mathrm{HCl}$ in every milliliter of a $10 \%$ sucrose water. Specifically, each rat was provided with free access to food, a $10 \%$ sucrose water source, and a $10 \%$ sucrose water adulterated with $.5 \mathrm{mg}$ of morphine. Measures of body days. weight, food, and liquid intake were taken daily.

RESULTS AND DISCUSSION

The results are presented in Fig. 1. The ordinate indicates the mean daily solution ingestion per $\mathrm{S}$ in milliliters. It is slear from Fig. 1 (left frame) that all three groups virtually ignored the water and maintained a high level of sucrose morphine intake. Analysis of variance indicated that the preference for sucrose morphine over water was statistically reliable for all three groups $(F=64.88, \mathrm{df}=1 / 8, \mathrm{p}<.01)$. There seems to be an intake hierarchy based on the sucrose content of the solutions. That is, over the 13-day period, the $10 \%$ sucrose morphine was ingested most while the $2.5 \%$ sucrose morphine was ingested least. However, these apparent differences were not statistically significant.

Consideration of the data presented in the left frame of Fig. 1 points up, at the very least, the utility of the sucrose adulteration procedure in production of high voluntary ingestion of morphine solution by the rat. Thompson \& Ostlund (1965) observed that their rats ingested an average of only $32.5 \mathrm{ml}$ of a $.5 \mathrm{mg} / \mathrm{ml}$ of morphine water solution, even after some 60 days of forced drinking. Therefore, adulterating the morphine solution with $10 \%$ sucrose increases the intake by nearly $300 \%$. One obvious limitation of the sucrose adulteration procedure is that rats are very good caloric meterers (Khavari, 1970). However, our observations indicate no undesirable effects on the

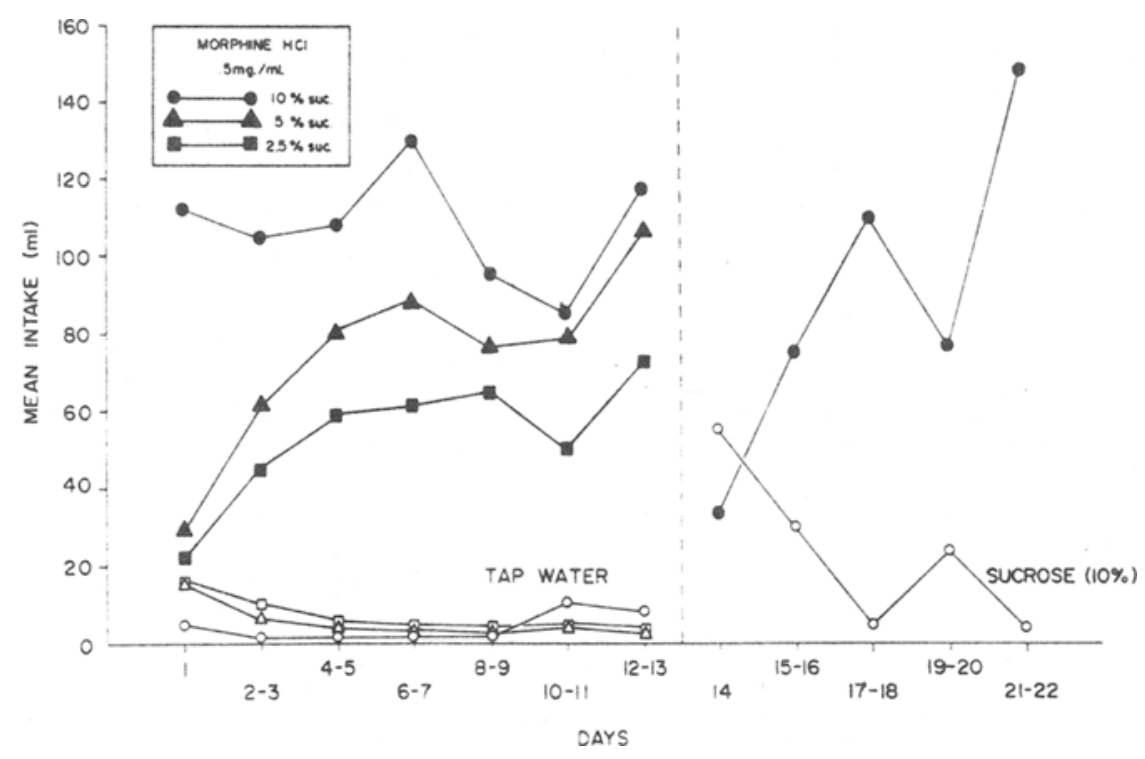

Fig. 1. Left: Three groups of rats maintained on sucrose-adulterated morphine for 25 days ingested greater quantities of the solution than of water, during a 13-day choice test. Right: The highest sucrose concentration group was presented with sucrose morphine and the sucrose vehicle for an additional 9 
rats' dietary regulation with sucrose concentrations as high as $10 \%$.

The data presented in the right frame of Fig. 1 provide clear evidence for the development of sucrose morphine preference over the vehicle. During Day 14 of the choice test (the first day of opportunity for the rats to choose between $10 \%$ sucrose and $10 \%$ sucrose containing $.5 \mathrm{mg} / \mathrm{ml}$ of morphine $\mathrm{HCl}$ ), the rats ingested liquid from both sources. During Day 14 the Ss consumed $32.7 \mathrm{ml}$ of sucrose morphine and $58.2 \mathrm{ml}$ of the vehicle, on the average. However, beginning with Day 15 and continuing through Day 22, all Ss showed a clear preference for the morphine sucrose over the vehicle $(F=10.54, \mathrm{df}=1 / 3$, $\mathrm{p}<.05$ ).

Clearly, there are a number of advantages and limitations associated with the oral procedure. Some of the advantages are: (1) the economic nature of the method, i.e., tedious and time-consuming injection and venous cannulation procedures are not necessary; (2) massive numbers of animals can be placed under this regimen with minimal care and effort requiring professional personnel; (3) the animals regulate their own intake of morphine by ingesting appropriate quantitites of the solution. A possible disadvantage is that rats are excellent in measuring their daily caloric intakes. Therefore, there is an upper limit for sucrose concentration that can be employed to boost morphine intake. Noncaloric flavoring may serve as effective substitutes for sucrose. In our laboratory, we have found that hypotonic saline $(.6 \%)$ is also a good morphine vehicle, but not as good as $10 \%$ sucrose. Also, both the peak and the maintenance levels of morphine achieved by the oral procedure may be different from those obtained via injection of equivalent doses. Nonetheless, the above oral procedure appears to be a highly effective and economical method for studying various physiological and psychological parameters of morphine dependence.

\section{REFERENCES}

KHAVARI, K. A. Some parameters of sucrose and saline ingestion. Physiology \& Behavior, 1970, 5, 663-666.

SCHUSTER, C. R., \& THOMPSON, T. Self administration and behavioral dependence on drugs. Annual Review of Pharamacology, 1969, 9, 483-502.

STOLERMAN, I. P., \& KUMAR, R. Preference for morphine in rats: Validation of an experimental model of dependence. Psychopharmacologia, 1970, 17, 137-150.

THOMPSON, T., \& OSTLUND, W., JR. Susceptibility to readdiction as a function of the addiction and withdrawal environments. Journal of Comparative \& Physiological Psychology, 1965, 60, 388-392. 